NIST Technical Note 2034

\title{
Defining 'kind of quantity'
}

\author{
David Flater
}

This publication is available free of charge from:

https://doi.org/10.6028/NIST.TN.2034

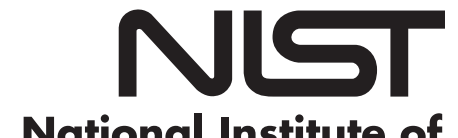

National Institute of

Standards and Technology

U.S. Department of Commerce 
NIST Technical Note 2034

\title{
Defining 'kind of quantity'
}

\author{
David Flater \\ Software and Systems Division \\ Information Technology Laboratory
}

This publication is available free of charge from:

https://doi.org/10.6028/NIST.TN.2034

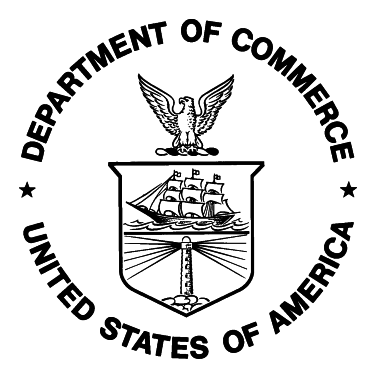

U.S. Department of Commerce

Wilbur L. Ross, Jr., Secretary

National Institute of Standards and Technology

Walter Copan, NIST Director and Undersecretary of Commerce for Standards and Technology 
Certain commercial entities, equipment, or materials may be identified in this document in order to describe an experimental procedure or concept adequately. Such identification is not intended to imply recommendation or endorsement by the National Institute of Standards and Technology, nor is it intended to imply that the entities, materials, or equipment are necessarily the best available for the purpose.

National Institute of Standards and Technology Technical Note 2034 Natl. Inst. Stand. Technol. Tech. Note 2034, 7 pages (February 2019) CODEN: NTNOEF

This publication is available free of charge from: https://doi.org/10.6028/NIST.TN.2034 


\title{
Defining 'kind of quantity'
}

\author{
David Flater
}

2019-02-06

\begin{abstract}
The definition of 'kind of quantity' given in the International Vocabulary of Metrology (VIM), 3rd edition, does not cover the historical meaning of the term as it is most commonly used in metrology. Most of its historical meaning has been merged into 'quantity,' which is polysemic across two layers of abstraction. I propose a model of 'kind of quantity' that is compatible with its historical meaning and distinguishable from 'quantity in a general sense.'
\end{abstract}

\section{Preface}

In this report I follow the convention that was provided in [1]:

Terms referring to terms themselves are delimited by double quotes (e.g. "measurement" is an 11-letter word); terms referring to concepts are delimited by single quotes (e.g. 'measurement' is a key concept of metrology); finally, terms with their usual referents are not delimited (e.g. measurement is a key process of metrology).

Double quotes are also used for verbatim quotations included inline. Bold and italic fonts are used or retained where necessary for consistency with the text of the International Vocabulary of Metrology (VIM) [2] and other cited references.

\section{Introduction}

The concept 'kind of quantity' has historically served to explain when and how it makes sense to compare two quantities, put them in order of magnitude, or add them together. Broadly speaking, these operations are meaningful when the quantities are of the same kind, otherwise not.

As important as this concept is, many scientists remain uncomfortable with its definition, particularly how to distinguish it from other ways of categorizing quantities. In this report I propose a model of 'kind of quantity' that makes it compatible with its historical meaning and distinguishable from closely related concepts such as 'quantity in a general sense.'

The remaining sections are organized as follows. Section 2 reviews the evolution of definitions of 'kind of quantity' in standards and related work. Section 3 explains the meanings of elements of the model. Section 4 provides the model itself. Section 5 continues with additional discussion of the model. Finally, Section 6 concludes. 


\section{History of "kind of quantity" and related terms}

Maxwell referred to kinds of quantities in his preliminary text on the measurement of quantities, writing "There must be as many different units as there are different kinds of quantities to be measured" [3].

An ontology by René Dybkaer provides a chronological list of relevant definitions appearing from 1951 through 2007 [4, Table 6.5]. In it, we can see an apparent schism between 'kind of quantity' seen as an abstraction, generalization, category, collection, or class of quantities and 'kind of quantity' seen instead as an element of information or aspect that quantities would have. A representative example of the former, as translated from DIN 1313 [5] by Dybkaer, is: "(Größenart) collection of quantities which are considered to be qualitatively alike and for which it is meaningful to add quantity values, independent from a quantity system to which they may belong." A representative example of the latter is the VIM 3 definition: "aspect common to mutually comparable quantities."

'Kind of quantity' was the primary organizing concept of the reference Quantities and Units in Clinical Chemistry (Recommendations 1966 et seq.) [6, 7]. Its descendant, the "silver book" [8], is organized by quantity dimensions first, then by kinds of quantities. Thus it is evident that the term has remained in continuous use in clinical chemistry from 1966 to the present day.

The first release of the VIM [9] distinguished 'quantities in a general sense' from 'specific quantities' and stated that "Quantities which are mutually comparable may be grouped together into categories of quantities." The 1987 corrections to VIM 1 added text to say that quantities that are mutually comparable are "also called quantities of the same kind."

The second edition of the VIM [10] replaced "specific quantity" with "particular quantity" (Def. 1.1 Note 1 and Def. 1.7) and replaced "mutually comparable" with "can be placed in order of magnitude relative to one another" (Def. 1.1 Note 2).

The third edition of the VIM [2] defined 'kind of quantity' as "aspect common to mutually comparable quantities," and it replaced the entire discussion of general versus particular quantities and categories of quantities with "The generic concept 'quantity' can be divided into several levels of specific concepts, as shown in the following table..." The examples in the lowest level are referred to in passing as "individual quantities."

Mari [11] performed an analysis of the VIM's 'kind of quantity' and related concepts using an object-oriented viewpoint. Dybkaer [12] rejected Mari's analysis, emphasizing that the VIM must be understood in the context of International Organization for Standardization (ISO) 704 [13] and ISO 1087-1 [14], standards for terminology work that are referenced by the VIM. [12] proceeded to explain that 'kind of quantity' occupies the role that $[13,14]$ refer to as "criterion of subdivision."

[1] used the terms "general quantity" and "individual quantity" where VIM 2 used "quantity in a general sense" and "particular quantity." [12] also used "individual quantities." In the following sections I will use "general quantity" and "individual quantity" consistently with [1]. Its further subdivision of "individual quantities' into 'quantities of objects' (which are called "addressed quantities" in [7]) and 'values of quantities' is not needed for this discussion.

\section{Model preliminaries}

It is not my intention to advocate for either concept-oriented or object-oriented viewpoints or language. What is important is that the necessary vocabulary for conceptual modelling is established, and for most purposes either type of language would do. Table 1 provides a cross-reference for the approximately equivalent notions from the concept-oriented ISO $704+1087-1$, the object-oriented Unified Modeling Language (UML) [17 $]^{1}$, and generic set theory.

\footnotetext{
${ }^{1}$ Although ISO/IEC 19501:2005 (UML version 1.4.2) might be superseded by ISO/IEC 19505-1:2012 and 19505-2:2012 (UML version 2.4.1), the older version contains a simple glossary of the terms that are relevant here [17, p. 411] while the newer version does not.
} 
Table 1: Approximate equivalences between concept-oriented, object-oriented, and set-theoretic language.

\begin{tabular}{|c|c|c|c|}
\hline ISO $704+1087-1$ & UML & Generic set theory & Notes \\
\hline Object & Object & Object & $\begin{array}{l}\text { "Objects are perceived or conceived; are abstracted or } \\
\text { conceptualized into concepts" [13]. Other names for ob- } \\
\text { jects include individuals, atoms, and ur-elements }[15,16] \text {. }\end{array}$ \\
\hline Concept & Class & Set & $\begin{array}{l}\text { "Concepts depict or correspond to objects or sets of ob- } \\
\text { jects" [13] }\end{array}$ \\
\hline (see notes) & Instance & Member / element & $\begin{array}{l}\text { "An object is an instance of a class" [17]; "single object } \\
\text { in an extension" [12]. "Instance" is used in passing in } \\
{[13, \S 6.4 .3] \text { and was adopted in [12] for use with ISO } 704} \\
+1087-1 \text {. }\end{array}$ \\
\hline Generic concept & Superclass & Superset & $\begin{array}{l}\text { "The superordinate concept in a generic relation" [13]; } \\
\text { "The generalization of another class" [17] }\end{array}$ \\
\hline Specific concept & Subclass & Subset & $\begin{array}{l}\text { "The subordinate concept in a generic relation" [13]; } \\
\text { "The specialization of another class" [17] }\end{array}$ \\
\hline (no & Metaclass & Set (of sets) & "A class whose instances are classes" [17] \\
\hline
\end{tabular}

When an object-oriented modelling language is used as a representation for conceptual modelling, a class represents a concept. ${ }^{2}$ Coincidentally, $[13, \S 5.1]$ uses the word "class" while explaining the nature of concepts: "Through observation and a process of abstraction called conceptualization, objects are categorized into classes, which correspond to units of knowledge called concepts, which are represented in various forms of communication (object $\rightarrow$ concept $\rightarrow$ communication)."

Regardless of the language used, the formal structure described below is maintained. However, to simplify the text, I will provide only the terms from the UML column of Table 1; for example, writing only "class" where one might instead write "concept/class/set."

A class has both intent and extent (or intension and extension). The intent consists of necessary and sufficient conditions to deduce that an object is an instance of the class. The extent consists of those objects that are instances. For example, the intent of the class 'bat' is provided by the definition of this subclass of mammals, while the extent is all of the bats in the world.

The intent of a subclass consists of the intent of its superclass (which it "inherits") plus at least one additional, narrowing condition. For example, the intent of 'bat' begins with being a mammal and then adds further conditions such as having wings. The extent of a subclass cannot include any object that is not in the extent of its superclass; usually, it will exclude some of them. Thus, as one navigates "downward" from most generic to most specific, intents expand and extents shrink.

If an object is an instance of a class, then it necessarily is an instance of every superclass of that class. For example, if 'mammal' is a subclass of 'animal,' 'bat' is a subclass of 'mammal,' and Gus is an instance of 'bat,' then it can be deduced that Gus is an instance of 'mammal' and 'animal' as well. For a concise representation, one explicitly states (or draws) only the instantiation relationships to the most specific applicable subclasses (Gus is an instance of 'bat').

A class may be directly related to any number of superclasses. A commonly used example is 'bat' being a subclass of both 'mammal' and 'winged animal,' both of which in turn are subclasses of 'animal.' The graph structure formed by generalization/specialization relationships therefore is a lattice rather than a tree.

The structure of metaclasses and their relationships to classes are completely analogous to the structure of classes and their relationships to objects. Only the layer of abstraction is different.

\footnotetext{
${ }^{2}$ The interpretation made in [12] that "a class is the extension of a concept" is inconsistent with common conceptual modelling practice and leads to confusion. As used here, a class represents the entirety of a concept, including both its intension and its extension.
} 


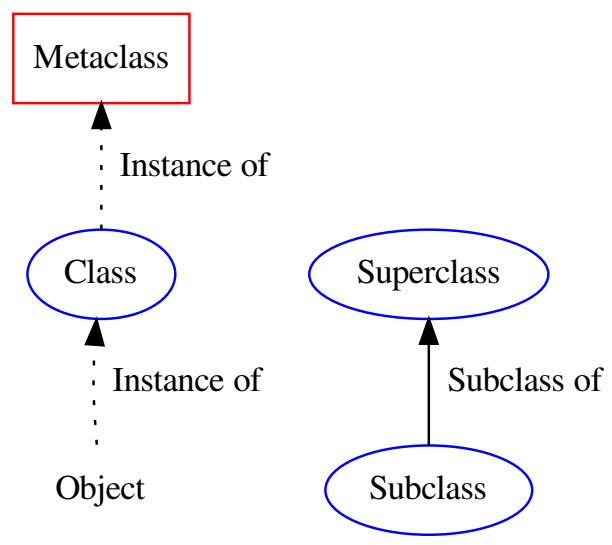

Figure 1: Guide to conceptual modelling notation used herein.

\section{Model}

The conceptual modelling notation to be used is introduced in Figure 1. Instantiation is represented with dotted arrows; specialization (subclassing) is represented with solid arrows. Classes and metaclasses are represented with blue ovals and red boxes respectively surrounding the name of the class or metaclass. Objects are represented without any surrounding shape.

Although the model is simple, it spans three layers of abstraction. ISO/International Electrotechnical Commission (IEC) 19502:2005, the Meta-Object Facility (MOF) Specification [18], identifies the three relevant layers with the shortened names M0 through M2. M0, also called the data, object, information, or instance layer, contains the objects. M1, also called the model or ontology layer, contains the concepts or classes that are abstractions over the contents of M0. M2, also called the metamodel or language layer, contains metaclasses that are abstractions over the contents of M1.

Using the MOF's names for the three relevant layers of abstraction, Figure 2 and Figure 3 express the model with examples that focus on illustrating 'kind of quantity' and 'general quantity' respectively. Individual quantities exist in M0. Classes of individual quantities, including the most general class of all individual quantities, exist in M1. 'Kind of quantity' and 'general quantity' exist in M2. They are metaclasses whose instances are classes of individual quantities. 'Kind of quantity' is a specialization of 'general quantity' that adds the narrowing condition of mutual comparability.

This model yields the following definitions:

A general quantity is a class of individual quantities.

A kind of quantity is a class of individual quantities that are mutually comparable.

Mutual comparability of individual quantities that are measured on a ratio scale [19] means that it is meaningful to place them in order of magnitude relative to one another and to add them together. If they are measured on an ordinal scale, then mutual comparability means only that it is meaningful to place them in order of magnitude relative to one another. Comparisons of nominal properties might be limited to testing for equality, but could extend to characterizing generic-specific relationships in a shared type system.

\section{Discussion}

A kind of quantity is distinguished from a general quantity by the added constraint that its instances must be mutually comparable. Examples of general quantities whose instances are not always mutually comparable are provided by a Note 6 in VIM 3: "The concept 'quantity' may be generically divided into, e.g., 'physical quantity,' 'chemical quantity,' and 'biological quantity,' or base quantity and derived quantity." 


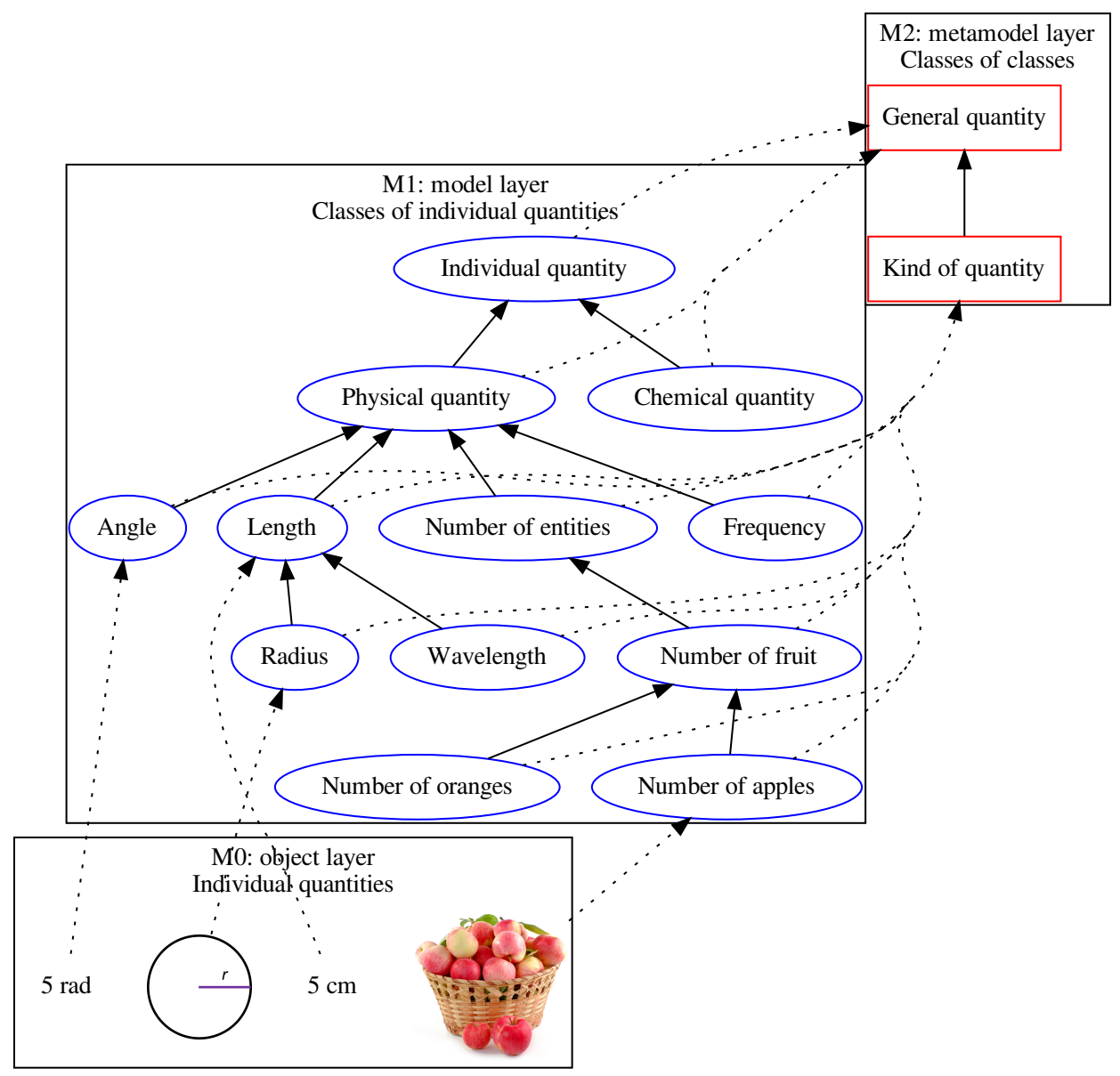

Figure 2: Conceptual model focusing on 'kind of quantity.'

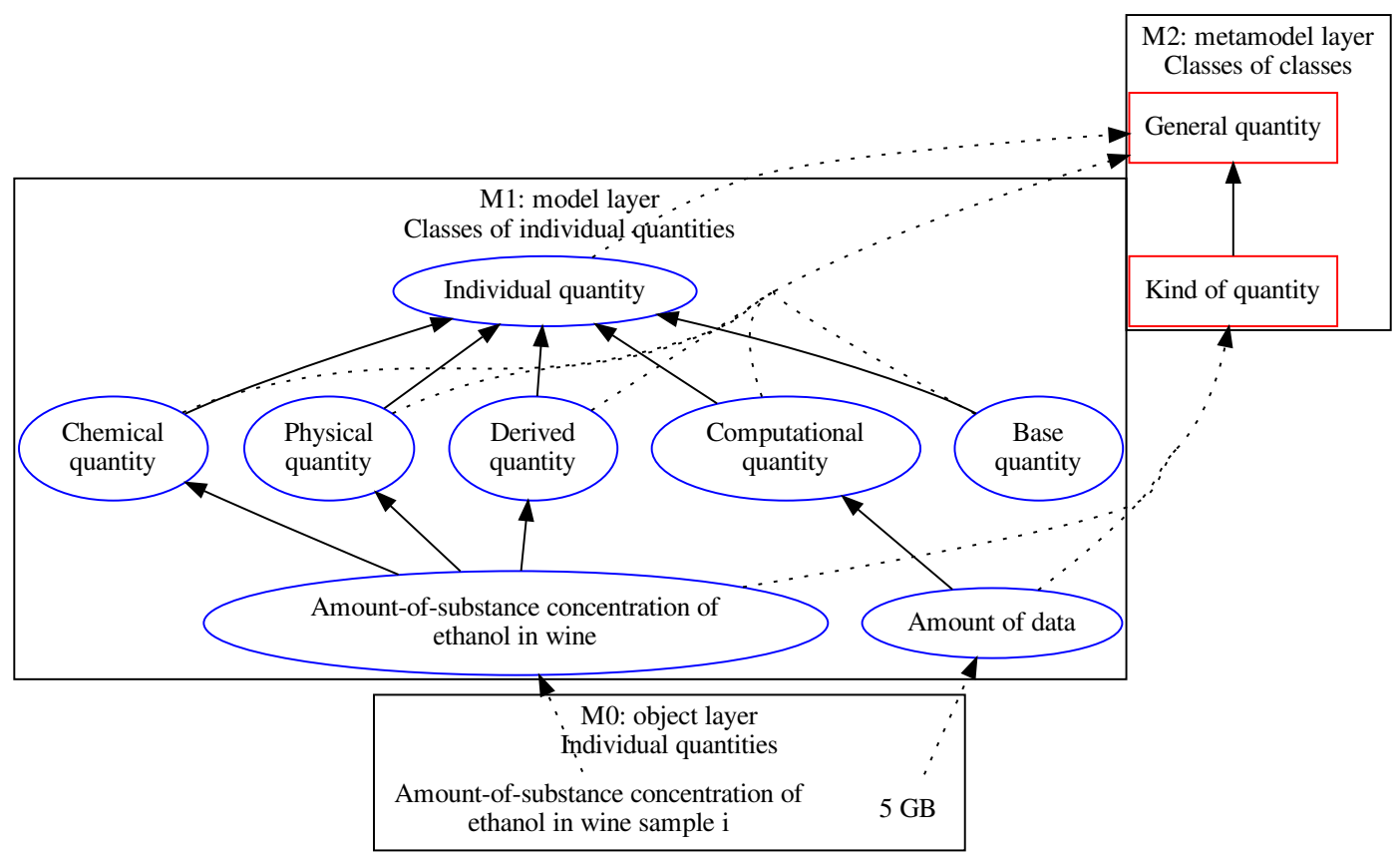

Figure 3: Conceptual model focusing on 'general quantity.' 
"Class of individual quantities" is not equivalent to the VIM's "aspect" or [12]'s "division criterion." A criterion or aspect might form part of the intensional definition of a class or metaclass, but it cannot be the same thing as the class or metaclass that it thusly gives rise to.

In practice, the use of 'kind of quantity' has mostly been consistent with its definition as a metaclass even in sources that cite the VIM's definition. For example, if we refer to the VIM definition, then the vernacular "amount-of-substance concentration is a kind-of-quantity" ought to be restated as "amount-of-substance concentration has a delimiting characteristic from kind-of-quantity" [12, §8]. But if 'kind of quantity' is a metaclass, the vernacular can be understood as saying that 'amount-of-substance concentration' is an instance of 'kind of quantity.' This seems like a clearer and more direct formalization of the intended meaning of the informal statement.

It is important to understand that comparability is not a simple dichotomy. Two quantities that are initially of different kinds can be comparable in a more general sense. For example, a wavelength and a radius are comparable only if their classifications are first generalized to length. To generalize them and add them together is to declare that the differences of kind between a wavelength and a radius are immaterial for the purpose at hand. Whether it is appropriate to do this is not an aspect of the individual quantities but rather depends on the context of use.

The terms themselves (the specific words used to refer to the concepts) matter only with respect to historical consistency and whatever unintended reactions they might elicit. Readers have expressed discomfort with "kind of quantity" because its English connotation is vague and the French translation "nature de grandeur" is unnatural to use, but no direct replacement has yet been proposed. What has herein been called "general quantity" could just as well be called "category of quantities" or "quantity class."

\section{Conclusion}

I hope that the model presented will be helpful in clarifying 'kind of quantity.' 'Kind of quantity' provides a more specific classification than 'quantity dimension.' For discussing which operations in quantity calculus are meaningful, there is no substitute for it.

Defining 'kind of quantity' and 'general quantity' as proposed requires three layers of abstraction. Since the M2 layer is not addressed in [13, 14], expressing this model in the form of the VIM could be problematic. The goal is to define a metaconcept that "depicts or corresponds to" a set of concepts, rather than a set of objects $[13, \S 0.2]$. My suggestion is to add definitions of the form proposed in Section 4, beginning "class (or category) of individual quantities...," and then reuse examples that appear in the notes under 'quantity.'

\section{Acknowledgments}

Thanks to Charles Ehrlich, Barbara Guttman, and Ben Long of the U.S. National Institute of Standards and Technology (NIST), and to Luca Mari of Università Carlo Cattaneo (LIUC), for providing comments and feedback. Additional thanks to Charles Ehrlich for supplying the text of the first edition of the VIM.

The opinions expressed in this report are the author's own and do not necessarily reflect the views or policies of NIST or the United States Government.

\section{References}

[1] Luca Mari, Charles Ehrlich, and Leslie Pendrill. Measurement units as quantities of objects or values of quantities: a discussion. Metrologia, 55(5):716-721, 2018. https://doi.org/10.1088/1681-7575/ aad8d8. 
[2] Joint Committee for Guides in Metrology. International vocabulary of metrology-Basic and general concepts and associated terms (VIM), 3rd edition, 2008. Amended in 2012, JCGM 200:2012, http: //www.bipm.org/en/publications/guides/vim.html.

[3] James Clerk Maxwell. A Treatise on Electricity and Magnetism, volume 1. Clarendon Press, 1873. https://en.wikisource.org/wiki/A_Treatise_on_Electricity_and_Magnetism.

[4] René Dybkaer. An ontology on property for physical, chemical, and biological systems, 2009. https: //doi.org/10.1351/978-87-990010-1-9.

[5] Deutsches Institut für Normung (DIN). Größen (Quantities). DIN 1313, 1998. As translated and quoted in [4].

[6] René Dybkær and K. Jørgensen. Quantities and Units in Clinical Chemistry (including Recommendation 1966 of the Commission on Clinical Chemistry of the IUPAC and of the IFCC). Williams \& Wilkins Co., 1967.

[7] IUPAC/IFCC. Quantities and units in clinical chemistry (Recommendations 1978). Pure and Applied Chemistry, 51(12):2451-2479, 1979. Available at https://old.iupac.org/publications/pac/1979/ pdf/5112x2451.pdf.

[8] Georges Férard, René Dybkaer, and Xavier Fuentes-Arderiu. Compendium of Terminology and Nomenclature of Properties in Clinical Laboratory Sciences: Recommendations 2016. Royal Society of Chemistry, 2016. https://doi.org/10.1039/9781782622451.

[9] BIPM/IEC/ISO/OIML. International vocabulary of basic and general terms in metrology (VIM), 1st edition, 1984. Amended in 1987.

[10] ISO Guide 99:1993. International vocabulary of basic and general terms in metrology (VIM), 2nd edition.

[11] Luca Mari. On (kinds of) quantities. Metrologia, 46(3):L11-L15, 2009. https://doi.org/10.1088/ 0026-1394/46/3/N01.

[12] René Dybkaer. ISO terminological analysis of the VIM3 concepts 'quantity' and 'kind-of-quantity'. Metrologia, 47(3):127-137, 2010. https://doi.org/10.1088/0026-1394/47/3/003.

[13] ISO 704:2009, third edition. Terminology work-Principles and methods, 2009-11-01.

[14] ISO 1087-1:2000, first edition. Terminology work-Vocabulary-Part 1: Theory and application, 2000$10-15$.

[15] W3C Recommendation 11 December 2012. OWL 2 Web Ontology Language Primer (Second Edition). https://www.w3.org/TR/2012/REC-ow12-primer-20121211/.

[16] Wikipedia. Urelement, 2018. https://en.wikipedia.org/wiki/Urelement.

[17] ISO/IEC 19501:2005. Unified Modeling Language Specification Version 1.4.2. OMG document number formal/05-04-01.

[18] ISO/IEC 19502:2005. Meta-Object Facility (MOF) Specification Version 1.4.1. OMG document number formal/05-05-05.

[19] Stanley S. Stevens. On the theory of scales of measurement. Science, 103(2684):677-680, June 1946. https://doi.org/10.1126/science.103.2684.677. 